\title{
ESOFAGOGASTRECTOMIA COM LINFADENECTOMIA EM DOIS CAMPOS NO CÂNCER DO ESÔFAGO TORÁCICO
}

\author{
ESOPHAGOGASTRECTOMY WITH TWO-FIELD LYMPHADENECTOMY FOR CANCER \\ OF THE THORACIC ESOPHAGUS
}

\author{
Gerardo Cristino Gavarrete Valladares ${ }^{1}$; Luis César Bredt, ACBC-PR ${ }^{2}$; \\ Luiz Antonio Negrão Dias ${ }^{3}$; Zacarias Alves de Souza Filho, ECBC-PR ${ }^{4}$; \\ Flávio Daniel Saavedra Tomasich, TCBC-PR ${ }^{5}$ Osvaldo Malafaia, TCBC-PR ${ }^{6}$
}

\begin{abstract}
RESUMO: Objetivo: Avaliar as indicações, sobrevida e fatores prognósticos da esofagogastrectomia com linfadenectomia em dois campos no câncer do esôfago torácico. Métodos: Foram avaliados 111 pacientes retrospectivamente no período de janeiro de 1990 a dezembro de 2001 sendo 83 homens e 29 mulheres. A idade média dos pacientes foi 55,1 anos (variando entre 35-79). A linfadenectomia em dois campos foi parcial (Standard) em 34 pacientes(30,6\%) e ampliada em 77(69,4\%). Resultados: A média de linfonodos dissecados foi de 22,6(variando entre 4 e 50). A doença R0 ocorreu em 53 pacientes(47,7\%) a doença residual microscópica (R1) em $57(52,3 \%)$ e a doença residual R2 em um paciente $(0,9 \%)$. A recidiva ocorreu em 32 pacientes $(28,8 \%)$ sendo em sete $(6,3 \%)$ cervical, $17(15,3 \%)$ locorregional e $19(17,1 \%)$ sistêmica. A morbidade e mortalidade pós-operatória foram de $31,5 \%$ e $9 \%$ respectivamente, sem diferença significativa em relação á extensão da linfadenectomia mediastinal. A sobrevida global dos 111 pacientes em cinco anos foi de 48,4\%, sem diferença significativa na sobrevida em relação á extensão da linfadenectomia, porém, houve aumento significativo na sobrevida livre de doença a favor dos paciente submetidos a linfadenectomia mediastinal ampliada(p=0,01). A ausência de doença residual (R0), comprometimento linfonodal ( $\mathrm{pN}$ ) e o número de linfonodos comprometidos inferior a quatro, indicaram bom prognóstico. Conclusão: A esofagogastrectomia com linfadenectomia em dois campos apresentou um impacto positivo na taxa de sobrevida em cinco anos nos pacientes com câncer do esôfago torácico, particularmente em relação aos pacientes com ECIII. A linfadenectomia mediastinal ampliada aumentou significativamente a sobrevida livre de doença (Rev. Col. Bras. Cir. 2008; 35(6): 374-381).
\end{abstract}

Descritores: Esofagectomia; Excisão de linfonodo; Neoplasias esofágicas; Tórax

\section{INTRODUÇÃO}

O câncer do esôfago é considerado doença agressiva com péssimo prognóstico, principalmente por seu diagnóstico quase sempre tardio, em pacientes (geralmente) idosos; estadiamento pré-operatório freqüentemente inexato e tratamento controvertido ${ }^{1},{ }^{2}$. Mundialmente o carcinoma espinocelular representa $70 \%$ dos tumores do esôfago ${ }^{3}$, embora tenha havido aumento dramático na incidência de adenocarcinoma nos USA e alguns paises europeus.

As modalidades terapêuticas utilizadas para o tratamento do câncer do esôfago são: cirurgia, radioterapia e quimioterapia, isoladas ou combinadas; a cirurgia, entretanto, é a que apresenta mais controvérsias, com relação à abordagem que pode ser trans-hiatal ou transtorácica e com relação à extensão da ressecção, se deve ser feita linfadenectomia em dois ou três campos $^{4-9}$. Alguns cirurgiões consideram o câncer do esôfago uma doença sistêmica ${ }^{10,11}$ indicando desta forma operações chamadas Standard, sem linfadenectomia, através de uma abordagem trans-hiatal ou transtorácica. A sobrevida a cinco anos destas ressecções tem permanecido entre 20 a $25 \%{ }^{12}$ e a recidiva local entre 20 a $60 \%{ }^{13}$.

Uma ressecção R0, isto é ressecção macroscópica e microscópica completa do tumor é considerada pela maioria dos cirurgiões como a melhor chance de cura do câncer de esôfago ${ }^{8,14,15}$. Embora a esofagectomia para obter uma ressecção R0 ainda não tenha sido definida. Considerando que a maioria das metástases são linfáticas e microscópicas uma linfadenectomia sistemática é indispensável para se obter uma ressecção $\mathrm{R} 0^{16}$.

Uma esofagogastrectomia radical com linfadenectomia ampla do mediastino e do abdômen através de laparotomia e toracotomia direita tem sido a nossa conduta no tratamento do câncer do esôfago e o objetivo deste estu-

1. Chefe do Serviço de Cirurgia Torácica do Hospital Erasto Gaertner

2. Chefe do Serviço de Cirurgia Oncológica do Hospital de Câncer de Cascavel.

3. Mestre; Cirurgião Titular do Serviço de Aparelho Digestivo do Hospital Erasto Gaertner.

4. Professor Titular de Clínica Cirúrgica da UPFP e PUCPR.

5. Doutor; Cirurgião Titular do Serviço de Aparelho Digestivo do Hospital Erasto Gaertner.

6. Livre-Docente pela Universidade Federal do Paraná; Professor Titular de Cirurgia da UFPR; Diretor Científico do Colégio Brasileiro de Cirurgia Digestiva,; Coordenador do Instituto de Pesquisas Médicas e do Programa de Pós-Graduação Stricto Sensu (Mestrado e Doutrorado) em Princípios da Cirurga do Hospital Universitário Evangélico de Curitiba; Professor Titular de Cirurgia da Faculdade Evangélica do Paraná.

Recebido em 23/06/2008

Aceito para publicação em 02/09/2008

Conflito de interesses: nenhum

Fonte de financiamento: nenhuma

Trabalho realizado no Serviço de Cirurgia Torácica do Hospital Erasto Gaertner. 
do foi avaliar retrospectivamente as indicações, sobrevida e fatores prognósticos da esofagogastrectomia com linfadenectomia em dois campos no tratamento do câncer de esôfago torácico.

\section{MÉTODO}

No período entre janeiro de 1990 e dezembro de 2001, 1898 pacientes com câncer do esôfago foram atendidos pelo Serviço de Tórax do Hospital Erasto Gaertner, Curitiba, Paraná. 166 pacientes foram submetidos a algum tipo de ressecção cirúrgica. Destes, 111 pacientes portadores de carcinoma espinocelular ou adenocarcinoma primário do esôfago torácico foram submetidos à esofagogastrectomia e linfadenectomia em dois campos (abdominal e mediastinal) e que constitui a população em estudo. Foram excluídos 30 pacientes submetidos a esofagogastrectomia sem linfadedenectomia (Ivor-Lewis stardat) 21 pacientes com adenocarcinoma de cárdia, um paciente submetido à radioterapia neoadjuvante em outro serviço, dois pacientes submetidos à esôfagogastrectomia trans-hiatal e um com carcinossarcoma.

As características clínico e patológicas dos 111 pacientes estão representadas na tabela 1 .

O percentual da perda de peso foi avaliada de acordo com Blackburn et al ${ }^{17}$.

\section{Avaliação Pré-Operatória e Estadiamento}

Após anamnese e exame físico os pacientes foram submetidos à endoscopia digestiva alta com biopsia, radiografia de tórax, radiografia de esôfago, ecografia abdominal, hemograma, estudo da coagulação, exames bioquímicos, avaliação cardiológica e provas de função pulmonar. Tomografia axial computadorizada de tórax não foi utilizada de rotina, sendo indicada apenas quando havia dúvida da ressecabilidade. A broncofibroscopia foi realizada apenas nos paciente com tumor da porção superior ou média do esôfago.

Tabela 1 - Casuística em 111 pacientes.

\begin{tabular}{lcc}
\hline Características Clínico e Patológicas & \\
\hline Sexo & & \\
Masculino & 82 pacientes \\
Feminino & 29 pacientes \\
Idade & $35-79$ anos \\
Média & $55,1$ anos (D.P.: 9,1$)$ \\
Tipo Histológico & \\
Carcinoma Espinocelular & $106 \quad(95,5 \%)$ \\
Adenocarcinoma & $05 \quad(4,5 \%)$ \\
Localizador do tumor & & \\
Porção Superior & $05 \quad(4,5 \%)$ \\
Porção Média & $51 \quad(45,9 \%)$ \\
Porção Inferior & $55 \quad(49,5 \%)$ \\
Avaliação Perda Ponderal & & \\
Nutridos (sem perda ponderal) & 42 & $(37,8 \%)$ \\
Perda Significativa & $44 \quad(39,6 \%)$ \\
Perda Acentuada & $25 \quad(22,5 \%)$ \\
\hline
\end{tabular}

Os critérios de operabilidade incluíam: ausência de metástases a distância, ausência de doença cardiovascular, hepática ou renal grave, pacientes com volume expiratório forçado no primeiro segundo (VEF1) superior a 1,5 litros e ausência de DPOC e enfisema pulmonar grave.

\section{Procedimento Cirúrgico}

O procedimento cirúrgico adotado em $97(87,4 \%)$ pacientes com tumores de terço médio e inferior do esôfago seguiu os princípios da técnica preconizada por Wong ${ }^{18}$, que consiste numa esofagogastrectomia subtotal, iniciando-se por laparotomia mediana supra umbilical com liberação da grande curvatura gástrica e ligadura dos vasos gastroepiplóicos esquerdos e gástricos curtos. Linfadenectomia do tronco celíaco com ligadura dos vasos gástricos esquerdos na sua origem. Os linfonodos do hilo hepático e ao longo da artéria esplênica também foram removidos. A manobra de Kocher foi realizada quando necessário seguida de piloromiomectomia ${ }^{19} \mathrm{e}$ jejunostomia a Witzel com posterior síntese da cavidade abdominal por planos. O paciente é posicionado em decúbito lateral esquerdo, procedendo-se à toracotomia póstero-lateral direita e abertura da cavidade torácica na altura do quinto espaço intercostal com ressecção do arco da veia ázigo, pleura parietal bilateral, com exceção dos casos de tumores superficiais, em que a pleura contra-lateral é preservada. $\mathrm{O}$ ducto torácico foi ressecado em toda sua extensão até o diafragma e incluído no espécime. A linfadenectomia mediastinal inclui os linfonodos paraesofageanos inferiores ao longo da aorta, diafragmáticos, subcarinais. Também os linfonodos recorrenciais esquerdos e paratraqueais direitos foram ressecados. O esôfago foi dissecado até o ápice do tórax e seccionado proximalmente; o estômago tracionado para dentro do tórax e a arcada da pequena curvatura foi seccionada $\mathrm{e}$ ligada distalmente à ligadura da artéria gástrica esquerda retirando-se a peça cirúrgica. Uma anastomose esofagogástrica intratorácica término lateral com grampeador intraluminal número 25 ou 29 (ILS Ethicon Ltd) foi realizada e a pequena curvatura gástrica suturada em dois planos. A anastomose foi mecânica em 94 pacientes e manual em três pacientes.

Para pacientes com tumores supracarinais (1/3 superior), a técnica utilizada é a preconizada por Akiyama et $a l^{20} \mathrm{e}$ foi realizada em 14 pacientes. A operação inicia com toracotomia direita, sendo o procedimento cirúrgico exatamente o mesmo descrito acima com secção e sutura do esôfago distalmente. A cavidade torácica é fechada e o paciente é posicionado em decúbito dorsal para laparotomia mediana supra-umbilical com liberação gástrica, linfadenectomia do tronco celíaco, abertura de hiato esofágico e tração do coto esofágico com estômago para fora da cavidade abdominal. Uma piloromiomectomia e manobra de Kocher são realizadas. A seguir o estômago é seccionado confeccionando-se um tubo gástrico. Realiza-se cervicotomia esquerda ao longo do bordo anterior do músculo esternocleidomastoídeo, o esôfago cervical é dissecado e junto com o tumor e tecidos linfáticos é exteriorizado e seccionado proximalmente. O tubo gástrico é tracionado até o pescoço pelo mediastino posterior ou pelo espaço retroesternal sendo realizada anastomose esofagogástrica manual em um plano. 


\section{Linfadenectomia em dois campos}

A partir de janeiro de 1990 iniciou-se a experiência com linfadenectomia em dois campos para tratamento cirúrgico dos tumores do esôfago torácico que consistiu de linfadenectomia abdominal; linfadenectomia mediastinal, que de acordo com Bumm et al ${ }^{7}$ caracteriza a linfadenectomia Standard e que nós denominamos de parcial. Posteriormente com a familiarização com a técnica, passou-se a realizar a linfadenectomia da cadeia paratraqueal e apical direita; caracterizando a linfadenectomia mediastinal ampliada; a cadeia recorrencial esquerda foi ressecada de rotina a partir de 1999.

\section{Estadiamento Patológico}

O estádio patológico de cada paciente foi obtido, utilizando as normas de classificação TNM da União Internacional de Contra o Câncer-UICC ${ }^{21}$. A doença residual (R) também foi avaliada utilizando a classificação RO para indicar ausência de tumor residual, R1 presença de tumor residual microscópico e $\mathrm{R} 2$ presença de tumor residual macroscópico ${ }^{22}$.

\section{Recidiva}

A recidiva local foi definida como qualquer recidiva tumoral na anastomose ou campo cirúrgico de dissecção mediastinal ou abdominal (locorregional). A recidiva cervical indicou aparecimento de metástases no pescoço. A recidiva a distância indicou aparecimento de metástases em outros órgãos.

\section{Terapia Neoadjuvante eAdjuvante}

Neste grupo de pacientes, quimioterapia neodjuvante com cisplatina e 5-fluoracil foi utilizada pelo Serviço de Oncologia Clinica, em pacientes com tumores localmente avançados em que havia dúvida da ressecabilidade. Dos 111 pacientes, $14(12,6 \%)$ foram submetidos à quimioterapia neoadjuvante e encaminhados para o tratamento cirúrgico. A quimioterapia adjuvante foi indicada em pacientes com comprometimento linfonodal extenso e realizada em nove pacien- tes $(8,1 \%)$. A radioterapia adjuvante foi indicada em quatro pacientes $(3,6 \%)$, dois com margem cirúrgica comprometida (um com doença residual macroscópica e um com metástases paratraqueais extensas). Dois pacientes $(1,8 \%)$ foram submetidos à radioterapia neoadjuvante.

\section{Análise Estatística}

Os dados coletados foram digitados em um banco de dados, utilizando o programa "Microsoft Access 7.0" para registro das informações, que foram analisadas estatisticamente com a utilização do "Epi-info" versão 2000. Os gráficos foram gerados utilizando a planilha eletrônica "Microsoft Excel 7.0”. Inicialmente realizou-se a análise descritiva das variáveis para verificar suas freqüências. Para estudo das variáveis consideradas qualitativas, foi utilizado o cálculo da porcentagem e as correlações foram realizadas utilizando o qui-quadrado em tabelas de contingência de dupla entrada $2 X 2$. Sempre que necessário devido às freqüências esperadas inferiores a cinco ou ao fato de ser pequena a amostra analisada, o teste do quiquadrado foi substituído pela prova não paramétrica de Fisher, a fim de se evidenciar distorções na apreciação da significância estatística. A sobrevida foi avaliada pela análise de sobrevida actuarial de Kaplan Méier calculadas com o programa Epi-info versão 2000. Foram considerados como casos não censurados (morte) todos aqueles que morreram em decorrência do câncer do esôfago, aqueles que morreram de outra causa não relacionada com á neoplasia, como morte pós-operatória e morte no domicilio sem evidência de doença em atividade. Foram considerados censurados todos os pacientes que estavam vivos na ocasião do ultimo contato e aqueles com perda de seguimento. $\mathrm{O}$ cálculo de significância foi realizado através do método de log rank e Breslow. Nível de significância para todos os testes foi $\mathrm{p}<0,05$.

\section{RESULTADOS}

\section{Complicações Pós-Operatórias}

As complicações pós-operatórias ocorreram em 31,5\% dos pacientes e estão demonstradas na tabela 2 .

Tabela 2 - Complicações Pós-Operatórias.

\begin{tabular}{lcc}
\hline Complicações Pós-Operatórias & Pacientes (N) & $\%$ \\
\hline Complicações Pulmonares Infecciosas & 21 & 18,9 \\
Complicações Pulmonares Não Infecciosas (DPOC, atelectasia, IRA) & 16 & 14,4 \\
Quilotorax & 1 & 0,9 \\
Empiema & 3 & 2,7 \\
Drenagem hemorrágica abundante & 6 & 5,4 \\
Infarto do Miocárdio, Arritmia Cardíaca, Choque Cardiogênico & 6 & 6,3 \\
Tromboembolismo Pulmonar & 1 & 0,9 \\
Lesão do Nervo Laríngeo Recorrente & 3 & 2,7 \\
Deiscência de Anastomose Esofágica & 7 & 6,3 \\
Intra-torácica & 2 & 1,8 \\
Cervical & 5 & 4,5 \\
Infecção de Ferida Operatória & 4 & 3,6 \\
Sepsis & 6 & 5,4 \\
Óbito Pós-operatório & 10 & 9 \\
\hline
\end{tabular}


Nos pacientes submetidos à linfadenectomia ampliada a taxa de complicações pós-operatórias foi de $35,5 \%$ e naqueles submetidos à linfadenectomia parcial, foi de 22,9\%, sendo que esta diferença não foi estatisticamente significativa $(\mathrm{p}=0,90)$. A mortalidade pós-operatória, definida como morte ocorrida em até 30 dias após a operação, foi de $9 \%$ (10 pacientes). A mortalidade pós-operatória nos pacientes submetidos à linfadenectomia ampliada foi de $10,5 \%$ e na linfadenectomia parcial foi de 5,7\% , não havendo significância estatística $(\mathrm{p}=0,77)$. As causas de óbito pós-operatório foram fístula de anastomose e sépsis em três pacientes, infecção pulmonar e sepsis em um paciente, insuficiência respiratória aguda em cinco pacientes e choque hipovolêmico um paciente.

\section{Estadiamento Patológico (pTNM)}

O estadiamento patológico dos 111 pacientes foi realizado de acordo com as normas do estadiamento TNM da UICC (1998) e está demonstrado na tabela 3.

Não houve nenhum tumor in situ. Em relação aos linfonodos, 54 pacientes $(48,6 \%)$ eram N0 e $57(51,4 \%)$ eram N1. Havia metástases linfonodais a distância em nove pacientes $(8,1 \%)$, correspondendo a M1a. 53 pacientes $(47,7 \%)$ doença residual RO, 57 (52,3\%), doença residual R1 e um paciente $(0,9 \%)$ apresentou doença residual R2. As margens cirúrgicas proximais estavam livres em $109(98,2 \%)$ e comprometidas em dois pacientes $(1,8 \%)$; não houve nenhum comprometimento da margem distal.

\section{Extensão da Linfadenectomia Mediastinal}

Dos 111 pacientes, 34 (30,6\%) foram submetidos à linfadenectomia mediastinal parcial (Standard) e $77(69,4 \%)$ a linfadenectomia mediastinal ampliada. A média de linfonodos

Tabela 3 - Freqüência de pacientes de acordo com o estadio patológico TNM (UICC).

\begin{tabular}{lcc}
\hline Estadio & Pacientes (N) & Porcentagens \\
\hline I & 6 & $5,4 \%$ \\
IIA & 46 & $41,4 \%$ \\
IIB & 7 & $6,3 \%$ \\
III & 43 & $38,7 \%$ \\
IVA & 9 & $8,1 \%$ \\
Total & 111 & $100 \%$ \\
\hline
\end{tabular}

dissecados nos 111 pacientes foi de 22,6 ( $\mathrm{DP}=10,5)$, com mínimo de quatro e máximo de 50; a média de linfonodos comprometidos foi de 3,2 (DP=2,6) com mínimo de um e máximo de 12. A média de linfonodos mediastinais dissecados foi de 15,7 com mínimo de um e máximo de $44(\mathrm{DP}=8,9)$. A média de linfonodos dissecados de acordo com a linfadenectomia mediastinal está demonstrada na tabela 4.

\section{Comprometimento linfonodal de acordo com a loca-}

\section{lização do tumor}

Os linfonodos mediastinais estavam comprometidos em 43 pacientes $(38,7 \%)$ e os abdominais, em 31 (34,4\%). Dos 56 pacientes com tumores em porção média e superior, 22 $(39,3 \%)$ apresentaram metástases linfonodais mediastinais e $13(28,9 \%)$, metástases abdominais. Dos 55 pacientes com tumores na porção inferior, $21(38,2 \%)$ apresentaram metástases linfonodais mediastinais e 18 (40\%) metástases linfonodais abdominais. A porcentagem de linfonodos comprometidos de acordo com a localização do tumor primário esta demonstrada na tabela 5 .

\section{Recidiva}

32 pacientes $(28,8 \%)$ apresentaram recidiva tumoral. Sete pacientes apresentaram recidiva cervical, sendo que em dois $(2,4 \%)$ este foi o único local de recidiva; 17 pacientes $(15,3 \%)$ apresentaram recidiva local, sendo que em cinco $(5,8 \%)$, este foi o único local de recidiva; 19 pacientes apresentaram recidiva sistêmica. $\mathrm{O}$ tempo mediano de recidiva foi de 16 meses, variando entre 6 a 46 meses, com mais de 50\% delas ocorrendo dentro dos dois primeiros anos (20 de 32 pacientes). O tempo mediano de recidiva após ressecção $\mathrm{R} 0$ foi de 36 meses (variando de 16 a 46 meses) e após ressecção $\mathrm{R} 1$ foi de 11 meses (variando de 6 a 32 meses).

\section{Sobrevida}

Do total de pacientes, 90,1\% (100 pacientes) apresentaram seguimento normal, com um tempo médio de seguimento de 38,6 meses. A taxa de abandono do seguimento foi de $9,9 \%$ (11 pacientes). Até a data do termino de levantamento dos dados, 61 pacientes $(55,0 \%)$ estavam vivos e $50(45,0 \%)$ morreram, sendo que 10 pacientes $(9 \%)$ tiveram morte pósoperatória; 7 (6,3\%) tiveram morte livre de doença e 33 (29,7\%) morte por câncer do esôfago.

A sobrevida global dos 111 pacientes foi em dois anos de 69,1\% e em cinco anos de 48,4 (Gráfico 01).

Tabela 4 - Média de linfonodos dissecados de acordo com a extensão da linfadenectomia mediastinal.

\begin{tabular}{|c|c|c|c|c|}
\hline \multirow[t]{2}{*}{ Sub-Grupos } & \multicolumn{2}{|c|}{ LinfadenectomiaAmpliada } & \multicolumn{2}{|c|}{ Linfadenectomia Parcial } \\
\hline & Média & DP & Média & DP \\
\hline Total de linfonodos & 24,9 & $(10,6)$ & 17,5 & $(8,5)$ \\
\hline Paratraqueal & 7,1 & $(6,7)$ & - & - \\
\hline Subcarinal & 5,7 & $(4,9)$ & 4,4 & $(2,7)$ \\
\hline Paraesofageano & 6,9 & $(5,2)$ & 6,5 & $(4,3)$ \\
\hline Perigástrico & 7,8 & $(5,8)$ & 7,9 & $(7,4)$ \\
\hline Tronco celíaco & 6,07 & $(3,9)$ & 3,7 & $(3,2)$ \\
\hline
\end{tabular}


Tabela 5 - Freqüência de metástases linfonodais de acordo com a localização do tumor.

\begin{tabular}{|c|c|c|c|c|c|c|c|c|}
\hline \multirow[t]{2}{*}{ Localização } & \multicolumn{2}{|c|}{ Linfonodos Paratraqueais } & \multicolumn{2}{|c|}{ Linfonodos Subcarinais } & \multicolumn{2}{|c|}{ Linfonodos Paraesofageanos } & \multicolumn{2}{|c|}{ Linfonodos Abdominais } \\
\hline & $\begin{array}{c}\text { Casos } \\
\text { Dissecados }\end{array}$ & $\begin{array}{c}\text { Taxa de } \\
\text { Comprometidos } \\
\mathbf{p}=\mathbf{0 , 3 7}\end{array}$ & $\begin{array}{c}\text { Casos } \\
\text { Dissecados }\end{array}$ & $\begin{array}{c}\text { Taxa de } \\
\text { Comprometidos } \\
\text { p=0,67 }\end{array}$ & $\begin{array}{c}\text { Casos } \\
\text { Dissecados }\end{array}$ & $\begin{array}{c}\text { Taxa de } \\
\text { Comprometidos } \\
\text { p=0,17 }\end{array}$ & $\begin{array}{c}\text { Casos } \\
\text { Dissecados }\end{array}$ & $\begin{array}{c}\text { Taxa de } \\
\text { Comprometidos } \\
\text { p=0,18 }\end{array}$ \\
\hline Terço médio & & $8,5 \%$ & & $12,5 \%$ & & $28,6 \%$ & & $28,9 \%$ \\
\hline$n=56$ & $\mathrm{n}=47$ & $\mathrm{n}=4$ & $\mathrm{n}=48$ & $n=6$ & $\mathrm{n}=49$ & $\mathrm{n}=14$ & $n=45$ & $\mathrm{n}=13$ \\
\hline $\begin{array}{l}\text { Terço inferior } \\
n=55\end{array}$ & $n=30$ & $\begin{array}{l}13,3 \% \\
n=4\end{array}$ & $\mathrm{n}=52$ & $\begin{array}{l}11,5 \% \\
n=6\end{array}$ & $\mathrm{n}=48$ & $\begin{aligned} & 39,6 \% \\
n= & 19\end{aligned}$ & $n=45$ & $\begin{aligned} & 40 \% \\
n & =18\end{aligned}$ \\
\hline $\begin{array}{l}\text { Total } \\
n=111\end{array}$ & $\mathrm{n}=77$ & $\begin{array}{l}10,4 \% \\
n=8\end{array}$ & $\mathrm{n}=100$ & $\begin{aligned} & 12 \% \\
\mathrm{n} & =12\end{aligned}$ & $\mathrm{n}=97$ & $\begin{aligned} & 34 \% \\
\mathrm{n} & =33\end{aligned}$ & $\mathrm{n}=90$ & $\begin{aligned} & 34,4 \% \\
n= & 31\end{aligned}$ \\
\hline
\end{tabular}

A sobrevida em cinco anos de acordo com estadiamento foi no EC I de 66,6\%, EC IIA de 65,1\%, EC IIB de $62,5 \%$, EC III de 33,3\% e no EC IVA de $0 \%$. Sobrevida global dos pacientes tratados com cirurgia exclusiva foi de $56,97 \%$ (82 pacientes) e sobrevida global dos pacientes tratados com alguma forma de neoadjuvância ou adjuvância foi de 37,19\% (29 pacientes). (Gráfico 2)

Para calcular a sobrevida de acordo com a doença residual os pacientes configurados com R1 e R2 foram agrupados, por motivos de cálculo estatístico. No grupo de paciente R1 a sobrevida em dois anos foi de $49,8 \%$ e em cinco anos de $32,5 \%$, e no grupo R0 (sem doença residual) a sobrevida em

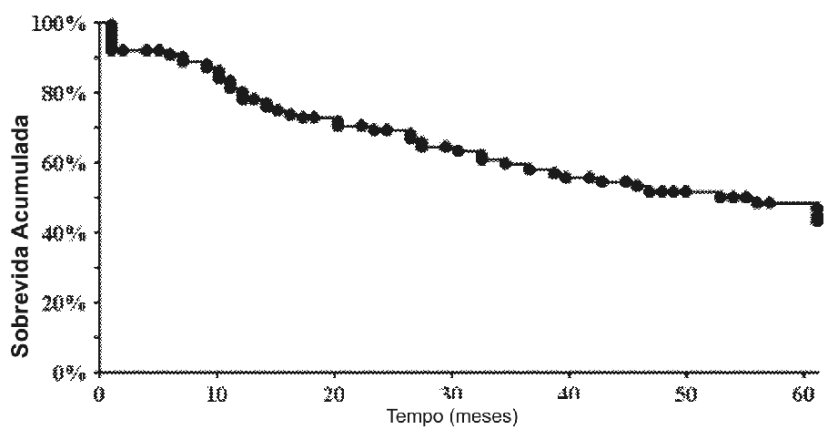

Gráfico 1 - Sobrevida global (Kaplan Meier).

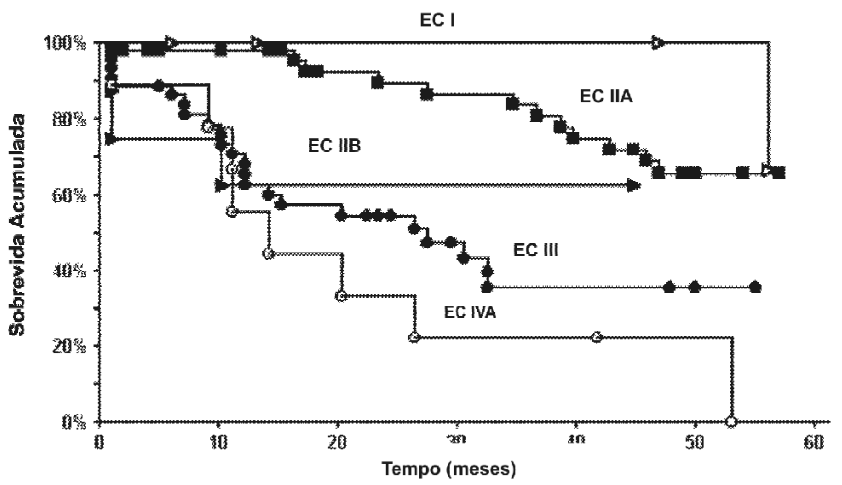

Gráfico 2 - Sobrevida de acordo com o estadiamento (PTNM). dois anos foi de $90,7 \%$ e em cinco anos de $66,3 \%$, sendo esta diferença significativa $(\mathrm{p}=0,0000)$. (Gráfico 03)

Nos pacientes com linfonodos negativos a sobrevida em cinco anos foi de $67,1 \%$ e naqueles com comprometimento linfonodal, foi de $31 \%$, sendo esta diferença significativa ( $\mathrm{p}=0,0000)$. (Gráfico 04)

No grupo de pacientes com um a quatro linfonodos comprometidos a sobrevida em dois anos foi de 57,8\% e em cinco anos de $42 \%$, no grupo com mais de quatro linfonodos comprometidos a sobrevida em dois anos foi de $23 \%$ e em cinco anos de $0 \%$. Nos paciente sem comprometimento linfonodal, a sobrevida em cinco anos foi de

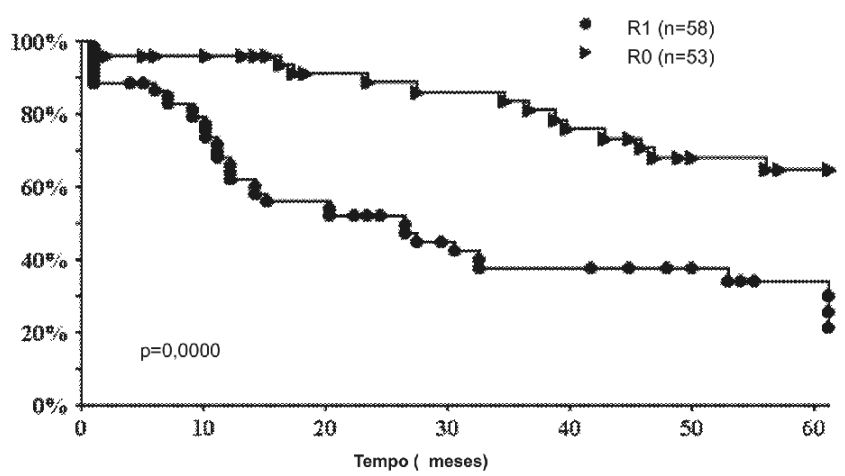

Gráfico 3 - Sobrevida global de acordo com a doença residual.

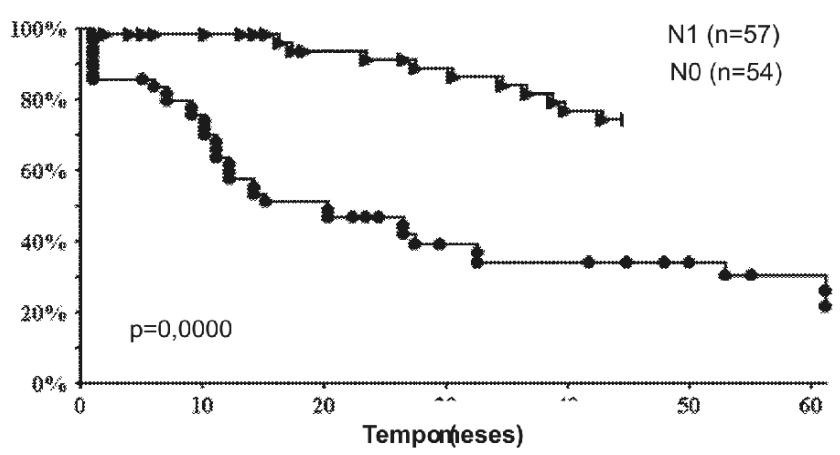

Gráfico 4 - Sobrevida de acordo com estadiamento PN. 
$67,1 \%$, sendo esta diferença significativa $(p=0,0000)$. (Gráfico 05)

A diferença, porém, na sobrevida em dois e cinco anos, não foi significativa quando um ou dois campos linfonodais estavam comprometidos. No grupo com um campo comprometido a sobrevida em dois anos foi de $46 \%$ e em cinco anos foi de $33 \%$. No grupo com dois campos comprometidos a sobrevida em dois anos foi de $48 \%$ e em cinco anos de $28 \%(\mathrm{p}=0,46)$.

Da mesma forma a sobrevida em dois e cinco anos não foi alterada pela extensão da linfadenectomia mediastinal. No grupo de pacientes submetidos à linfadenectomia parcial (Standard) a sobrevida em dois anos foi de $63,7 \%$ e em cinco anos de $40,3 \%$. No grupo de pacientes submetidos à linfadenectomia ampliada foi, em dois anos de $72,3 \%$ e em cinco anos de 53,2\% ( $\mathrm{p}=0,87)$, porém, a sobrevida livre de doença nos pacientes nos paciente submetidos à linfadenectomia parcial foi em dois anos de $61,7 \%$ e em cinco anos de 55,8\%; nos pacientes submetidos a linfadenectomia ampliada, foi em dois anos de $84,4 \%$ e em cinco anos, de $77,9 \%$, sendo esta diferença estatisticamente significativa $(\mathrm{p}=0,01)$. (Gráfico 06)

\section{DISCUSSÃO}

O papel da linfadenectomia para tumores gastrointestinais foi introduzido há mais de um século, porém o papel da linfadenectomia no câncer do esôfago permanece controverso ${ }^{4,6}$. Alguns cirurgiões ${ }^{10}$ consideram o câncer de esôfago uma doença sistêmica, portanto, a ressecção cirúrgica é apenas paliativa, alegando que os benefícios da linfadenectomia não têm sido comprovados, propiciando apenas um aumento da morbidade e mortalidade, e por isso indicando operações menos agressivas como a esofagectomia trans-hiatal. No outro extremo há outros cirurgiões, principalmente japoneses ${ }^{23-25}$, que indicam a ressecção radical com linfadenectomia em três campos, baseados na freqüência de metástases cervicais, mesmo em tumores superficiais, com a finalidade de reduzir as taxas de recidiva local e aumentar a sobrevida que de acordo com estes autores variou entre 55 e $68 \%$ em cinco anos.

Existe um grupo intermediário de cirurgiões ${ }^{4,7,26-28}$ que recomendam a linfadenectomia em dois campos, com menor

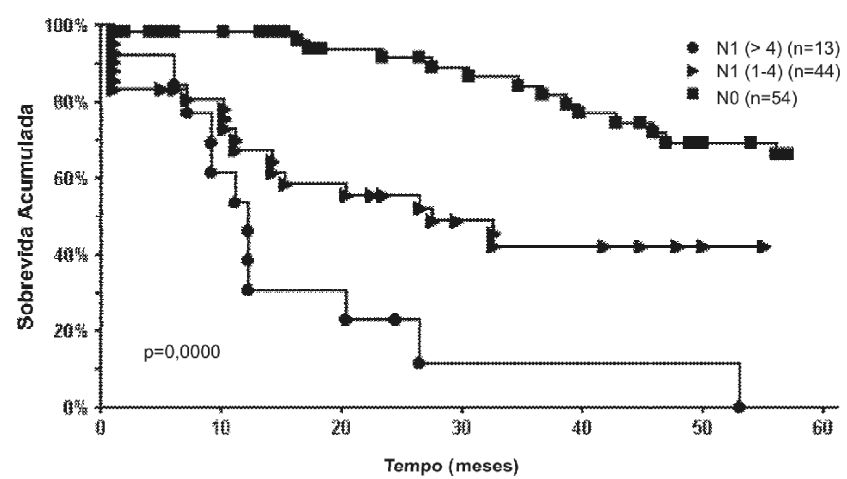

Gráfico 5 - Sobrevida de acordo com o número de linfonodos comprometidos. morbidade e mortalidade comparável, baseados no padrão de disseminação linfática e localização do câncer de esôfago, em que tumores infracarinais seguem a drenagem linfática distal e tumores supracarinais a drenagem linfática proximal; nestes últimos a linfadenectomia em três campos estaria indicada.

Os fundamentos da linfadenectomia são: primeiro, a disseminação linfática do câncer do esôfago é precoce e ampla, 28,6\% dos tumores que invadem a muscular mucosa e $54,1 \%$ dos tumores que invadem a submucosa apresentam metástase linfonodal ${ }^{24}$. Segundo, estadiamento patológico mais completo. Terceiro, diminuir a taxa de recidiva local e quarto, prolongar a sobrevida. De acordo com o "Consenso Sobre a Ressecção Cirúrgica para o Câncer do Esôfago Torácico", realizado em Milan em $1995^{\circ}$, pelo menos 15 linfonodos entre mediastinais e abdominais deveriam ser examinados pelo patologista. A média de linfonodos dissecados no presente estudo foi de 22,6 e a média de linfonodos mediastinais dissecados foi de 15,7; demonstrando que nosso estadiamento é confiável e reforça a indicação de linfadenectomia mediastinal.

A recidiva local varia entre 20 a $60 \%$ em ressecções Standard ${ }^{13}$. Enquanto que com esofagectomia e linfadenectomia em dois campos esta taxa tem variado entre 8 a $25 \%{ }^{13,29-32}$. No presente estudo a taxa de recidiva local foi de $15,3 \%$, inferior às obtidas com ressecções limitadas.

Os resultados da linfadenectomia em três campos têm mostrado que o comprometimento dos linfonodos cervicais varia de $27,4 \%$ a $46,6 \%{ }^{24,33,34}$, portanto, era de se esperar uma taxa de recidiva cervical correspondente nas ressecções em que não é realizada a linfadenectomia cervical, porém, os estudos sobre o padrão de recidiva após esofagectomia transtorácica e transhiatal têm verificado que a taxa recidiva cervical varia de $3,6 \%$ a $11 \%{ }^{30-32,35}$. No presente estudo a taxa de recidiva cervical foi de $6,3 \%$, indicando que poucos pacientes se beneficiariam da linfadenectomia cervical.

Consideramos importante observar o beneficio significativo na sobrevida em cinco anos nos paciente com ECIII, que nesta série foi de $33,3 \%$ e na literatura varia entre 10 a $17 \%$ com ressecções Standard e entre $27 \%$ a $39 \%$ com ressecções radicais $^{12,13}$. O comprometimento linfonodal, sabidamente é um dado indicativo de mau prognóstico, não entanto existe um grupo de pacientes com metástases linfonodais nos quais é possível aumentar a sobrevida com ressecção radical. O

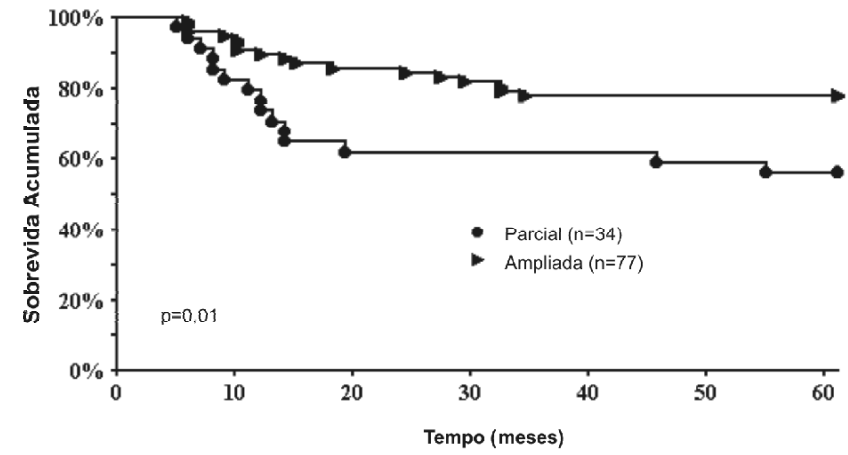

Gráfico 6 - Sobrevida livre de doença de acordo com a extensão da linfadenectomia mediastinal. 
número de linfonodos comprometidos tem sido citado por vários autores como um dos melhores indicadores de prognóstico ${ }^{24,36,37}$. Nesta série a presença de mais de quatro linfonodos comprometidos indicou pior prognóstico e nenhum paciente sobreviveu cinco anos, enquanto que no grupo de pacientes com um a quatro linfonodos comprometidos a sobrevida em cinco anos foi de $42 \%$.

A ressecção R0 implica na exérese do tumor primário com margem de segurança proximal, distal e lateral por meio de ressecção de estruturas adjacentes e da linfadenectomia. A previsão de uma ressecção R0 depende da localização do tumor primário e do estadiamento, tornado improvável a sua realização em tumores supracarinais transmurais, sendo possível somente em tumores T1 e T2 ${ }^{8,27}$. Nossos resultados mostram que o grupo de pacientes sem doença residual (R0) apresentaram uma sobrevida em cinco anos significativamente melhor que os pacientes com doença residual microscópica; embora, nesta série $73 \%$ dos tumores eram T3 (transmurais), 95\% dos tumores estavam localizados na porção média e inferior do esôfago o que permitiu a realização de ressecções com margem de segurança.

A taxa de comprometimento linfonodal no mediastino $(38,7 \%)$ e abdominal $(34,4 \%)$ foi similar, indicando a mesma tendência de comprometimento, independente da localização do tumor. A extensão da linfadenectomia mediastinal, entretanto não aumentou a sobrevida em cinco anos, mas houve um aumento significativo da sobrevida livre de doença nos pacientes submetidos à linfadenectomia ampliada; desta for- ma acreditamos como outros autores ${ }^{38}$, que a linfadenectomia mediastinal superior proporciona um estadiamento mais completo e deve prevenir a recidiva nesta região, reforçando nossa conduta atual, já que não houve diferença significativa na morbidade nem na mortalidade entre a linfadenectomia parcial e ampliada.

A sobrevida global deste grupo de pacientes foi de $69,1 \%$ em dois anos e de 48,4\% em cinco anos, mostrando resultados superiores aos obtidos com ressecções limitadas, que de acordo com Altorki et al ${ }^{12}$ varia entre 17 e $29 \%$ em cinco anos, e comparável com as taxas de sobrevida com linfadenectomia em dois campos que varia entre $26,2 \%$ e $54,9 \%$ 4,28.

No presente estudo, 29 pacientes foram submetidos à tratamento neodjuvante ou adjuvante por doença localmente avançada, e a sobrevida global neste grupo de pacientes foi de $37,19 \%$.

A ressecção continua sendo o tratamento primário do câncer do esôfago potencialmente curável e a ressecção R0 deve ser o principal objetivo. A abordagem e a extensão da ressecção permanecem controvertidas e na ausência de estudos randomizados, os dados da literatura são difíceis de interpretar. Até o presente momento, a associação de radioquimioterapia neoadjuvante e adjuvante não têm demonstrado vantagem na sobrevida sobre a ressecção isolada. Assim com Kitajama et al ${ }^{39}$, consideramos pouco provável que as taxas de sobrevida de $51 \%$ em cinco anos ${ }^{40}$ obtidas com esofagectomia e linfadenectomia radical, poderão ser superadas com a ressecção isolada.

\begin{abstract}
Background: To evaluate the indication, survival and prognostic factors of esophagogastrectomy with two-field lymphadenectomy in thoracic esophageal carcinoma. Methods: From January 1990 to December 2001, 111 patients were retrospectively analyzed.There were 83 men and 29 women.Median age was 55,1 years (range 35-79).Partial( standard) mediastinal lymphadenectomy was performed in 34 patients (30,6\%) and extended lymphadenectomy in 77(69,4\%). Results:The median number of dissected lymph node was 22,6(range 4-50).No residual disease(R0) was found in 53 patients $(47,7 \%)$, microscopic residual disease $(R 1)$ in $57(52,3 \%$ and macroscopic residual disease $(R 2)$ in $1(0,9 \%)$. Recurrence occurred in 32 patients $(28,8 \%)$ : cervical in 7(6,3\%), locoregional in 17(15,3\%) and distant in 19(17,1\%).Operative morbity and mortality were 31,55 and $9 \%$ respectively with no significant difference between partial(standard) and extended mediastinal lymphadenectomy. The overall 5 year survival rate was 48,4\%, with no significant diference with respect to the extension of the mediastinal lymphadenectomy. However there was a significant diference in disease-free survival rate in favor of patientes who underwent extended mediastinal lymphadenectomy $(0,01)$.No residual disease $(R O)$, no regional lymph node involvement $(p N O)$ and number of positive lymph node less than 4 were identified as good prognostic factors. Conclusion: The esophagogastrectomy with two-field lymphadenectomy had a positive impact in 5-year survival rate in patients with thoracic esophageal carcinoma particularly in ECIII patients. Extended mediastinal lymphadenectomy improved the disease-free survival.
\end{abstract}

Key words: Esophagectomy; Lymph node excision; Esophageal neoplasms; Thorax.

\section{REFERÊNCIAS}

1. Lerut T, Coosemans W, De Leyn P, Deneffe G, Topal B, Van de Ven C, Van Raemdonck D. Reflections on three field lymphadenectomy in carcinoma of the esophagus and gastroesophageal junction. Hepatogastroenterology. 1999; 46(26):717-25.

2. Lerut T, Coosemans W, De Leyn P, Van Raemdonck D, Nafteux $\mathrm{P}$, Moons J. Optimizing treatment of carcinoma of the esophagus and gastroesophageal junction. Surg Oncol Clin N Am. 2001; 10(4):863-84, $\mathrm{x}$

3. Gamliel Z. Incidence, epidemiology, and etiology of esophageal cancer. Chest Surg Clin N Am. 2000; 10(3):441-50.

4. Siewert JR, Stein HJ. Lymph-node dissection in squamous cell esophageal cancer - who benefits? Langebecks Arch Surg. 1999; 384(2): 141-8.

5. Altorki NK. Three-field lymphadenectomy for esophageal cancer. Chest Surg Clin N Am. 2000; 10(3):553-60. 
6. Law S, Wong J. What is appropriate treatment for carcinoma of the thoracic esophagus? World J Surg. 2001; 25(2):189-95.

7. Bumm R, Wong J. More or less surgery for esophageal cancer: extent of lymphadenectomy in esofagectomy for squamous cell esophageal carcinoma: how much is necessary? Dis Esophagus. 1994; 7:151-6.

8. Fumagalli U. Resective surgery for cancer of the thoracic esophagus: result of a consensus conference held at the $6^{\text {th }}$ World Congress of the International Society for Diseases of the Esophagus. Dis Esophagus. 1996; 9(Suppl 1):30-8.

9. Blom D, Peters LH, DeMeester TR. Controversies in the current therapy of carcinoma of the esophagus. J Am Coll Surg. 2002; 195(2):241-50.

10. Orringer MB. Occult cervical nodal metastases in esophageal cancer: preliminary results of three-field lymphadenectomy [editorial]. J Thorac Cardiovasc Surg. 1997; 113(3):538-9.

11. Müller JM, Erasmi H, Stelzner M, Zieren U, Pichlmaier H. Surgical therapy of oesophageal carcinoma . Br J Surg. 1990; 77(8):845-57.

12. Altorki NK. The rationale for radical resection. Surg Oncol Clin N Am. 1999; 8(2):295-305

13. Altorki N, Skinner D. Should en bloc esophagectomy be the standard of care for esophageal carcinoma. Ann Surg. 2001; 234(5):581-7.

14. Siewert JR, Roder JD. Lymphadenectomy in esophagel cancer surgery. Dis Esophagus. 1992; 5(1): 91-7.

15. Stein HJ, Fink U, Bartels H, et al. More or less surgery for esophageal cancer: who benefits neoadjuvant therapy of esophageal cancer? Dis Esophagus. 1994; 7:156-61.

16. Nishimaki T. An appropriate esophagectomy for esophageal cancer: a lack of evidence and growing disparity between Western and Eastern standards [editorial]. Ann Thorac Cardiovasc Surg. 2006; 12(4):231-3.

17. Blackburn GL, Bistrian BR, Maini BS, Schlamm HT, Smith MF Nutritional and metabolic assessment of the hospitalized patient. JPEN. 1997; 1(1): 11-22.

18. Wong J. Stapled esophagogastric anastomosis in the apex of the right chesr after subtotal esophagectomy for carcinoma. Surg Gynecol Obstet. 1987; 164(6): 568-72.

19. Bains MS. Ivor Lewis esophagectomy. Chest Surg Clin N Am. 1995; 5 (3):515-26.

20. Akiyama H, Hiyama M, Hashimoto C. Resection and reconstruction for carcinoma of the thoracic oesophagus. Br J Surg. 1976; 63(3):206-9.

21. Brasil. Ministério da Saúde. Secretaria de Assistência à Saúde. Instituto Nacional do Câncer. TNM: classificação de tumores malignos. Rio de Janeiro: INCa; 1998.

22. Hermanek P. pTNM and residual tumor classifications: problems of assessment and a prognostic significance. World J Sug. 1995;19(2):184-90.

23. Kato H, Tachimori Y, Mizobuchi S, Igaki H, Ochiai A. Cervical, mediastinal, and abdominal lymph node dissection (three-field dissection) for superficial carcinoma of the thoracic esophagus. Cancer. 1993; 72(10):2879-82.

24. Akiyama H, Tsurumaru M, Udagawa H, Kajiyama Y. Radical lymph node dissection for cancer of the thoracic esophagus. Ann Surg. 1994; 220(3): 364-72; discussion 372-3.

25. Nishimaki T, Suzuki T, Kanda T, Obinata I, Komukai S, Hatakeyama K. Extended radical esophagectomy for superficially invasive carcinoma of the esophagus. Surgery. 1999;125(2):142-7.

26. Law S, Wong J. Two-field dissection is enough for esophageal cancer. Dis Esophagus. 2001; 14(2):98-103.

27. Law S, Wong J. Current Management of esophageal cancer. J Gastrointest Surg. 2005; 9(2):291-310.
28. Watanabe H, Kato H, Tachimori Y. Significance of extended systemic lymph node dissection for thoracic esophageal carcinoma in Japan. Recent Results Cancer Res. 2000; 155:123-33.

29. Tam PC, Siu KF, Cheung HC, Ma L, Wong J. Local recurrences after subtotal esophagectomy for squamous cell carcinoma. Ann Surg. 1987; 205 (2):189-94.

30. Law SY, Fork M, Wong J. Pattern of recurrence after oesophageal resection for cancer: clinical implications. Br J Sug. 1996; 83(1):107-11.

31. Dresner SM, Griffin SM. Pattern of recurrence following radical oesophagectomy with two-field lymphadenectomy. Br J Sug. 2000; 87(10): 1426-33.

32. Mariette C, Balon JM, Piessen G, Fabre S, Van Seuningen I, Triboulet JP. Pattern of recurrence following complete resection of esophageal carcinoma and factors predictive of recurrent disease. Cancer. 2003; 97(7): 1616-23.

33. Isono K, Sato H, Nakayama K. Results of a nationwide study on the tree-field lymph node dissection of esophageal cancer. Oncology. 1991; 48:411-20.

34. Altorki NK, Skinner DB. Occult cervical nodal metastasis in esophageal cancer: preliminary results of tree-field lymphadenectomy. J Thorac Cardiovasc Surg.1997; 113(3):5404.

35. Hulscher JB, van Sandick JW, Tijssen JG, Obertop H, van Lanschot JJ. The recurrence pattern of esophageal carcinoma after transhiatal resection. J Am Coll Surg. 2000; 191(2):143-8.

36. Nishimaki T, Suzuki T, Suzuki S, Kuwabara S, Hatakeyama K. Outcomes of extendend radical esophagectomy for thoracic esophageal cancer. J Am Coll Surg. 1998;186(3):306-312.

37. Rizk N, Venkatraman E, Park B, Flores R, Bains MS, Rusch V; American Joint Committee on Cancer staging system. The prognostic importance of the number of involved lymph nodes in esophageal cancer: implications for revisions of the American Joint Committee on cancer staging system. J Thorac Cardiovasc Surg. 2006; 132(6):1374-81.

38. Hirai T, Hihara J, Inoue M, Nishimoto N, Kagawa Y, Yoshida K, Toge T. Significance of lymph node dissection of the superior mediastinal region in esophageal cancer patients. Dis Esophagus. 2000; 13(3):198-202.

39. Kitajima M, Kitagawa Y. Surgical treatment of esophageal cancer-the advent of the era of individualization. N Engl J Med. 2002; 347(21):1705-9.

40. Altorki N, Kent M, Ferrara C, Port J. Three-field lymph node dissection for squamous cell and adenocarcinoma of the esophagus. Ann Surg. 2002; 236(2):177-83.

Como citar este artigo:

Valladares GC, Bredt LC, Dias LA, Malafaia O, Tomasich FD, Souza Filho ZA. Esofagogastrectomia com linfadenectomia em dois campos no câncer do esôfago torácico. Rev Col Bras Cir. [periódico na Internet] 2008; 35(6). Disponível em URL: http://www.scielo.br/ rcbc

Endereço para correspondência:

Gerardo Cristino Gavarrete Valladares ${ }^{1}$

Hospital Erasto Gaertner - CEPEP

A/C Patrícia Varella

Rua Ovande do Amaral, 201 - Jardim das Américas

81.520-060 - Curitiba - PR

E-mail: gerardo@iop.com.br

pvarella@erastogaertner.com.br 